\title{
Higher Order Encoding: Process or state?*
}

\author{
NEAL F. JOHNSON \\ Ohio State University, Columbus, Ohio 43210
}

\begin{abstract}
While prior data has seemed to suggest that learning occurs as a result of encoding information into higher order memory units, rather than the formation of interitem associations, the process whereby the encoding occurs has been left relatively unspecified. Two encoding models were outlined which differed to the extent that one assumed that encoding occurred as an active process after the specific items of information were registered in memory, while the other assumed that information is initially registered in memory in the encoded state. The results from two studies seem to offer the most support for the second of these two models.
\end{abstract}

During the past few years, there has been an increasing tendency to move away from traditional associative explanations of learning and descriptions of what is learned toward conceptions of the process that presume a somewhat more active organism. Traditional conceptions of learning were quite mechanistic and, to a large degree, the empirical effort was aimed at identifying the conditions under which an assumed atom of learning (i.e., an association) was established. The nature of the learning atom was more or less assumed and not subjected to empirical investigation, and, consequently, the explanatory adequacy of the construct was never seriously questioned. It was simply assumed that what Ss learned were two-place direct relationships, or associations, between observable items and that all knowledge states ultimately could be analyzed into compounds of such associations. Furthermore, an assumption, which was more or less implicit to the approach, was that to conceptualize the domain of learning in that manner would eventually lead to a more thorough understanding of the phenomenon than would conceptualizing it in any other manner.

An alternative approach is to view the learner as an active processor of information and attempt to understand learning through an understanding of the mechanisms and the processes whereby he acquires information. That is, rather than trying to understand the phenomenon of learning in terms of the conditions under which an assumed single type of relationship is acquired, it might be more profitable to try to define the mechanisms and processes the learner engages when he attempts to register information in memory. Furthermore, such an approach does not rest on any

*This article represents a revision of a paper presented at a symposium on Coding Processes in Human Memory at the 1972 meetings of the Midwestern Psychological Association in Cleveland, Ohio. The original title of the paper was "Organization and the coding view of what is learned." The work that is reported here was supported in part by Grants MH 08526 and MH 11236 of the National Institute of Mental Health, United States Public Health Service, and by Grant 534.1 from the Office of Science Information Service. National Science Foundation, to the Computer and Information Science Research Center, Ohio State University. rigid assumption of what is learned, but rather it leaves that issue open to empirical investigation. In fact, as will be noted below, one approach to examining the processing mechanisms engaged by a learner is to explore the nature of what has been learned.

\section{LEARNING AS ORGANIZATIONAL PROCESSES}

One conception of the learning process, which has implications for both the mechanisms whereby information is acquired and the issue of what is learned, is the hypothesis that learning is, in fact, an active process of organizing the to be learned material and then including the learned material within an existing organized network of information within memory. It is assumed that learning results from the coding processes entailed by the organization.

While the term organization implies some set of relationships, the nature of these relationships depends upon a particular conception of organization. For example, a Markov process might be viewed as an organizational net, and it would represent a linear conception of organization. An alternative model is to view the organization in terms of unitizing or chunking (Miller, 1956), with small item sets encoded into higher order units, which themselves can be encoded into yet higher order units, etc. For example, words can be encoded into phrase units, which in turn can be encoded into subject and predicate units, and, at a higher level. those units can be encoded into sentence units. It is this hierarchical conception of organization that will be used here, and a specific organization for a sequence can be defined by the hierarchical encoding pattern.

It is assumed that the unitization process, regardless of the level at which it occurs, is the establishment of a higher order memory code which is a single device that represents the information in the lower order codes (as opposed to some notion of a simple amalgamation of that information) (Johnson, 1970, 1972). Following from such a coding notion, one can operationally define a response unit or chunk as a response set that tends to be recalled or forgotten in an all or none manner. and the stage the unitization or encoding process has 
achieved can be assessed by the degree of that all or none tendency. This represents the major empirical implication of the coding conception of the unitization process.

There has been a great deal of support for such a notion of coding, beginning as early as the work of Müller in the late 1800s (Woodworth, 1938). More recently, the clustering effects in free recall (Bousfield, 1953; Shuell, 1968) have suggested that the items in response chunks tend to be both retrieved adjacently and forgotten in an all or none manner. The work on serial recall (Johnson, 1970, 1972; Bower \& Winzenz, 1969) also suggests a tendency toward all or none forgetting of response chunks, as well as a tendency for all or none retrieval of chunks from memory during learning. All of these effects seem quite consistent with a coding view of the unitization process.

It is important to note that, while the coding view of chunking implies a tendency for all or none recall and forgetting, it does not imply that the tendency should be complete. For example, early in learning only part of the information from a chunk may be included within the code. Furthermore, following learning, there is no reason why some of the information from a code could not be forgotten, leaving the other information unaffected. If the code is retrieved on a recall attempt, only the information it currently represents can be produced.

\section{PROCESS AND STATE CONCEPTIONS OF ENCODING}

It has been argued elsewhere (Johnson, 1970, 1972) that codes might be viewed as opaque containers in the sense of representing information, rather than being the information itself. If that is the case, then codes should not necessarily reflect the information they represent in any direct way. For example, while there is a two-thirds overlap in the information in the chunks SBJ and SXJ, there would be no reason to believe that the similarity should be reflected in their respective codes. If Ss were asked to learn a chunked sequence such as SBJ FQL ZNG and then asked to learn SXJ FQL ZNG, the learning of the new code for the first chunk during the second learning should retroactively interfere with the later recall of the first-chunk code from the first task. Recall of that code is measured by the Ss ability to recall the unchanged letters from that chunk, and the results indicate rather massive forgetting (Johnson, 1970). Furthermore, if the critical chunk has four letters, recall of the unchanged letters is low, and it is not influenced by whether there are one, two, or three changed letters. That is, degree of similarity, measured in terms of overlapping content, did not influence the effect. Data such as these seem to support the view of codes as opaque containers.

An interesting issue that emerges from these considerations concerns when and how the opaque code is established. For example, a chunk like SBJ might be initially registered in short-term memory as three distinct items which, as a result of some kind of active processing on the part of the $S$, are later encoded into a single opaque coding device that represents the entire set of three letters. That view of encoding would suggest that with repeated trials Ss attempt to include more and more information within codes that are established very early in learning. Such a conception of the process is consistent with views presented by Tulving (1968), Mandler (1967), and Johnson (1972).

An alternative view is to assume that the information is initially registered in memory as an encoded unit, and the content of the code might be viewed as a generative scheme that can be used for recovering the information it represents. Such a view suggests that on any learning trial the chunks in a study display are initially registered in memory as single opaque codes, and the specific items of information are not themselves ever separately registered in memory. For example, if SBJ was a chunk that either appeared alone or as one of a sequence of chunks, this view would indicate that the individual letters S, B, and $\mathrm{J}$ would not be separately represented in memory during their presentation on study trials, but rather some single opaque coding device would be registered that would represent those individual items and from which the items could be generated if the item information was needed. This view of encoding would suggest that it is an all or none process, and the function of repeated trials would be to allow the $S$ multiple opportunities to identify or discover an adequate code.

The hypothesis that individual items are initially registered in memory and that formation of the opaque code results from an active processing of these items by the learner was examined in an experiment similar to the retroactive interference study described above. The preceding study indicated that, if a chunk was well encoded by a $S$, then asking him to learn another chunk that differed from the first by only one letter was a sufficient condition for losing the entire first chunk from memory, even the common letters. If the first chunk was stored in memory as a collection of individual items, rather than encoded as an opaque code, then changing one letter in the chunk should result in a loss of that item, but no others. That is, if the chunk was not as yet unitized and there was no single code representing all the items, then interfering with the S's memory for one of the to be unitized items should have no influence on his memory for other items in that set. However, as the $S$ begins to process that item in the sense of encoding it with the other items in the set into a single opaque code, such interference with the item should result in an increasing tendency for all the items in the set to be lost from memory. For example, if a display such as SBJ FQL ZNG is initially registered in memory as nine individual items, then following that display immediately by SXJ FQL ZKG should result in a loss of $B$ and $N$, but $S, J, Z$, and $G$ should be retained as well as FQL. However, if the Ss are given time to actively encode the sequence into three opaque codes 
representing SBJ, FQL, and ZNG before the interfering sequence is presented, then there should be a greater tendency for $\mathrm{S}, \mathrm{J}, \mathrm{Z}$, and $\mathrm{G}$ to be lost from memory, relative to the loss for $\mathrm{FQL}$.

In order to examine that hypothesis, Ss were shown a nine-consonant sequence for $6 \mathrm{sec}$. The sequence was grouped as SBJ FQL ZNG. That was followed either 0 or $24 \mathrm{sec}$ later by a 6 -sec display of an interfering sequence that was the same as the first except that one letter in each of two chunks was changed (e.g., SXJ FQL ZKG). Then, $30 \mathrm{sec}$ after the termination of the first sequence (i.e., either 0 or $24 \mathrm{sec}$ after the second display terminated), they were asked to recall either the first or the second sequence. The Ss counted backward by threes during the retention intervals.

The results indicated that recall of the unchanged letters from changed chunks (S, J, Z, and $G$ ) was significantly below that of the letters in the unchanged chunk ( $F, Q$, and $L)$. Most important, however, was the fact that the magnitude of the effect was not influenced by the interval between the appearances of the first and second sequences. In addition, the magnitude of the influence of the first-sequence chunk on the retention of the second-sequence chunk (i.e., the proactive interference) also was not influenced by how long the $S$ had to process the first sequence before the point of interference. Both the retroactive and proactive interference effects in this study would seem to suggest that the unitization of the first sequence was as complete as it was going to be immediately after presentation and, hence, it is inferred that the encoding of the information into opaque codes was complete at that time.

The fact that Ss do not more completely encode chunks when they are given an opportunity to do so subsequent to the termination of an input does not rule out the hypothesis that such encoding is the result of an active process on the part of the S. It may be that the items in chunks are registered separately in memory at the onset of an input, and then during the input the items are encoded into an opaque code that represents the entire set. That assumes that the encoding process occurs only during the study interval. In that an antirehearsal activity (counting backward by threes) was used in the above study, that explanation of the data seems quite reasonable.

At this point, then, the issue is whether to be unitized item sets are initially registered in memory in their encoded states, or are the individual pieces of item information separately registered, followed by a rapid encoding process during presentation. The problem, of course, was to find a procedure that could be used to assess the degree of higher order encoding of a set of chunk-size materials at the time of initial registration. The procedure decided upon was again based upon the temporal implications of the encoding and decoding processes described above. The Ss were presented with a sequence of displays of three consonants each, and their task was to press one button indicating "yes" if a presented display was some predesignated target item (e.g., SBJ) and to press the other button if the display was not the target. In another condition, the Ss were presented with a similar sequence of three-consonant displays, but their task was to indicate whether the display contained a predesignated target letter (e.g., does SBJ contain S).

If Ss initially register chunks as individual items, which they later unitize by encoding them into a higher order opaque code, then they should be able to indicate that SBJ contained the letter S more rapidly than they could indicate SBJ was SBJ. However, if chunks are initially registered as opaque codes that only represent the items they contain, then Ss would have to decode the opaque encoding of SBJ before they would know that it contained an S. Therefore, if that was the case, they should be able to indicate that the display SBJ was the predesignated target SBJ more rapidly than they could indicate that the display contained the predesignated letter $S$.

The results of the study indicated that Ss were able to indicate that SBJ was SBJ about $70 \mathrm{msec}$ faster than they were able to indicate that it contained an $S$. The same procedure has been used in several studies where the displays were English words and the targets were either words or letters within words, and the results of such studies have been similar to the one with consonants. Word units can be identified about $100 \mathrm{msec}$ faster than letters within words. That is even true when the S's attention is drawn to one letter position. For example, Marmurek and Johnson (1973) asked Ss to respond "yes" only if the target letter was the first letter within a word. Furthermore, they were told that the target letter would never appear in any other position, and it did not. Even under these circumstances, the word-level units were identified about $70 \mathrm{msec}$ faster than the letters. It seems relatively clear from these studies that the $\mathrm{S}$ has unit-level information available in memory before he knows anything about the components of the unit. That would suggest that items within chunks are initially registered in memory as encoded units and, given that encoded state, to recover specific item information requires an active decoding of the opaque code on the part of the $S$.

As a final disclaimer, it is important to note that no assumption is made regarding the adequacy of the initial encoding in terms of its permanence, the extent to which the opaque code represents all the information it should represent, or that all the encoded information within a code is equally represented in terms of the permanence of the representation. That is, it might take multiple attempts to arrive at a completely adequate opaque code. if adequacy is defined in terms of complete availability of all the information over an extended period of time. 


\section{REFERENCES}

Bousfield. W. A. The occurrence of clustering in the recall of randomly arranged associates. Journal of General Psychology, 1953. 49, 229-240.

Bower, G. H., \& Winzenz, D. Group structure. coding. and memory for digit series. Journal of Experimental Psychology Monograph, 1969, 80(Pt. 2). 1-17.

Johnson, N. F. The role of chunking and organization in the process of recall. In $\mathrm{G}$. Bower (Ed.), The psychology of learning and motivation. Vol. 4. New York: Academic Press. 1970. Pp. 171-247.

Johnson, N. F. Organization and the concept of a memory code. In A. W. Melton and E. Martin (Eds.), Coding processes in human memory. Washington, D.C: Winston, 1972. Pp. 125-159.

Mandler, G. Organization and memory. In K. W. Spence and J. $\mathrm{T}$. Spence (Eds.), The psychology of learning and motivation.
Vol. 1. New York: Academic Press, 1967. Pp. 327-372.

Marmurek, H. H. C., \& Johnson. N. F. Identification of words and letters within words. Paper presented at the meeting of the Midwestern Psychological Association. Chicago. May 1973.

Miller. G. A. The magical number seven plus or minus two: Some limits on our capacity for processing information. Psychological Review, 1956.63, 81-97.

Shuell, T. J. Retroactive inhibition in free-recall learning of categorized lists. Journal of Verbal Learning \& Verbal Behavior, 1968. 7. 797-805.

Tulving. E. Theoretical issues in free-recall. In T. Dixon and D. Horton (Eds.), l'erbal behavior and general behavior theory. Englewood Cliffs. N.J: Prentice-Hall. 1968. Pp. 2-36.

Woodworth, R. S. Experimental psycholog: New York: Holt. 1938.

(Received for publication August 30. 1973.) 